\title{
REMINISCENCE, MOTIVATION, AND PERSONALITY
}

A Case Study in Experimental Psychology 


\title{
REMINISCENCE, MOTIVATION, AND PERSONALITY A Case Study in Experimental Psychology
}

\author{
H. J. Eysenck and C. D. Frith \\ University of London \\ London, England
}




\section{Library of Congress Cataloging in Publication Data}

Eysenck, Hans Jurgen, 1916-

Reminiscence, motivation, and personality.

Bibliography: $p$.

Includes index.

1. Memory, 2. Motivation (Psychology) 3. Personality. I. Frith, C. D., joint author. II. Title.

All rights reserved

No part of this book may be reproduced, stored in a retrieval system, or transmitted, in any form or by any means, electronic, mechanical, photocopying, microfilming, recording, or otherwise, without written permission from the Publisher 


\begin{abstract}
Although the invention of plausible hypotheses, independent of any connection with experimental observations, can be of very little promotion of natural knowledge; yet the discovery of simple and uniform principles, by which a great number of apparently heterogeneous phenomena are reduced to coherent and universal laws, must ever be allowed to be of considerable importance toward the improvement of the human intellect.
\end{abstract}

Thomas Young: First Bakerian Lecture, 1801 
This book is dedicated to the many friends, colleagues, and students whose work in this department contributed to our final formulations:
A. Broadhurst
J. Germain
R. Kessell
K. H. Star
P. Broadhurst
J. Grassi
P. Ley
K. Sterky
G. Claridge
M. Garg
A. E. Maxwell
C. Stroh
A. M. Clark
J. E. Gray
P. D. McLean
J. Tizard
D. Cookson
H. C. Holland
N. O'Connor
E. Treadwell
C. G. Costello
E. Howarth
J. Pinillos
A. Iseler
S. Rachman
W. Thompson
J. Das
A. Jensen
G. E. Sartory
O. Tunstall
W. DiScipio
H. Gwynne Jones
O. H. M. Seu
P. H. Venables
S. B. G. Eys
F. H. Farley
J. Jones
P. A. Slater
K. M. Warwick
M. P. Feldman
D. C. Kendrick
I. Spielman
O. White
G. Wilson 


\section{Contents}

Introduction $\ldots \ldots \ldots \ldots \ldots \ldots \ldots \ldots \ldots \ldots \ldots \ldots \ldots \ldots \ldots$

PART I THE ORIGIN OF THE GRAND DESIGN $\ldots \ldots \ldots \ldots$

Chapter 1 Kraepelin and the Age of Innocence ........... 3

Definition of Reminiscence .............. 3

The Specificity of Reminiscence ........... 6

Muscular Fatigue and Reminiscence .......... 11

Kraepelin's Studies of Reminiscence . . . . . . . . . 14

An Early Consolidation Theory ............. 33

Chapter 2 The Pursuit Rotor: An Apparatus for All

Occasions ...................... 37

Chapter 3 The Beginning of Investigations on a Grand

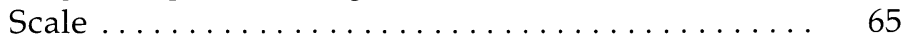

Chapter 4 The All-Purpose Apparatus Meets the All-

Purpose Theory ................. 97

PART II THE FAILURE OF THE GRAND DESIGN . . . . . . . . 129

Chapter 5 Reminiscence and Motivation ............. 131

Chapter 6 Post-Rest Upswing and Downswing ........... 165

Early Studies of Warm-up . . . . . . . . . . . . 166

Warm-up in Verbal Learning . . . . . . . . . . . . 169

Warm-up in Motor Skills ............... 172 
Is Upswing an Artifact of Measurement? . . . . . . 180

Measures of Performance Alternative to

Time-on-Target ................... 182

The Underlying Components of Rotary-Pursuit

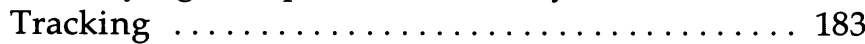

Conclusions .......................... 191

Chapter 7 Transfer of Training and Interpolated Activity ...... 199

Introduction .......................... 199

Bilateral Reminiscence .................... 202

Practice and Interpolated Activity at Different

Rotation Speeds.................... 207

Practice and Interpolation of Motor Tasks Other

than the Standard Pursuit Rotor ............ 213

Changes in the Distribution of Practice . . . . . . . 217

Conclusions ........................... 223

Chapter 8 Individual Differences in Ability as

Determinants of Performance and

Reminiscence ..................... 227

Chapter 9 Individual Differences: Extraversion . . . . . . . . 255

Chapter 10 Individual Differences: Schizophrenia . . . . . . . . . . 297

PART III THE NEW LOOK IN REMINISCENCE . . . . . . . . 321

Chapter 11 Consolidation: The Failure of Inhibition Theory . . . . . 323

The Need for a New Approach .............. 326

The Consolidation Theory of Memory .......... 327

How Does Consolidation Improve Performance? . . . 330

The Evidence for Action Decrement ........... 331

Evidence for Consolidation in Pursuit-Rotor

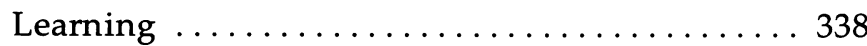

Consolidation in the Explanation of

Reminiscence and Other Phenomena of

Pursuit-Rotor Learning .................. 339

A One-Factor Theory of Reminiscence . ......... 344

Chapter 12 Strategies in Performance: What Is This Thing

Called Learning? ...................... 351

Problems in the Measurement of Pursuit-Rotor

Performance ......................... 352

Hits and Average Hit Lengths .............. 352

Rest Pauses and Responses . . . . . . . . . . . . . . 357

The Search for the Rest Pause .............. 360 
What Happens in between Responses? . . . . . . . . 364

What Is Learned in a Pursuit-Tracking Task? ...... 366 A Summary of This New Model of Pursuit

Tracking and Its Implications $\ldots \ldots \ldots \ldots \ldots 369$

Strategies of Performance ................. 373

Some Physiological Speculations............... 374

Chapter 13 The Rise and Fall of Reminiscence: An

Explanation Is Proposed and Some

Morals Are Drawn .................................... 377

Reminiscence in Tapping ................. 383

Reminiscence in Verbal Learning ............. 385

Reminiscence in Pursuit-Rotor Learning ....... 388

Epilogue Fifty Years of Pursuit-Rotor Studies .............. 393

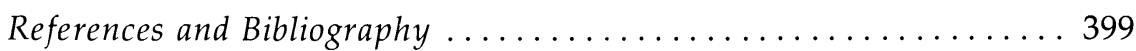

Reference Index ............................. 421

Subject Index ............................... 427 


\section{Introduction}

This is a book on reminiscence, or more modestly a book on reminiscence in motor tasks, or more modestly still on reminiscence in pursuit rotor learning, with occasional references to other types of reminiscence. The vast majority of experiments investigating reminiscence with the pursuit rotor have been carried out within the framework of Hullian learning theory. Thus, of necessity, this book also will be much concerned with that theory. Some readers may feel that so much detailed attention paid to one piece of apparatus and one now rather discredited theory, is overdone; we could not agree with such an evaluation.

There are several features of pursuit-rotor performance which make it particularly worthy of attention. One of the more important of these features is the easy replicability of many of the phenomena found in performance of this task; this is our first point. Replicability is the life blood of science; what cannot be replicated by any well-trained observer is of doubtful status in science, and on this score pursuit-rotor work certainly emerges as perhaps the most reliable set of observations in experimental psychology. The effects of massing and spacing; of rest pauses of different length; of switching from massed to spaced learning, or vice versa; of interpolating different activities; of introducing distracting stimuli; of switching from right to left hand, or vice versa; of changing the speed of rotation, or the diameter of the target diskthese are clear-cut and replicable as few phenomena in psychology are. There are few other examples where a young student can be told to carry out an experiment on a few subjects with the absolute certainty that the results will be predictable and precisely in line with what the literature says they ought to be; we should cherish such experiments, and hope that in due course there will be more of them! 
It may seem to some readers that we lay too much stress on reliability of findings, and their replicability. We do not believe this to be a fault, but rather a virtue. Having both had some training in the physical sciences, we are astonished that replication of important findings is so rare in psychology; in the hard sciences replication is regarded as an essential feature of advance. Even when attempts are made at replication in psychology, there are usually so many changes in apparatus, choice of subjects, and parameter values that what is regarded as a "replication" is in fact equivalent to a change in virtually all the important properties from the original study. Psychologists, to put it bluntly, lack only too frequently the essential research discipline which has been responsible for the great advances in physics and chemistry; the failure to agree on the physical dimensions and properties of the pursuit rotor, to which Ammons (1955) has drawn attention, is only one example of this defect. Where no two studies are alike in such properties of the apparatus as speed of rotation, size of target, height of working surface, length of stylus, material properties of rotating disk and target, maintenance, lighting, and many more, it is surprising, and speaks highly for the indestructibility of the main phenomena observed, that so much agreement has in fact been found. Where outcomes are less robust, as in verbal learning, these faults emerge with even greater clarity. Changes in research parameters which are intended, and made for theoretical reasons are of course acceptable and welcome; most changes found in the literature, however, are simply capricious or motivated by a vain desire to be different and "original." We have tried to follow our own advice; in important areas, such as the influence of personality or motivation on reminiscence, we have replicated our findings five or more times before accepting the conclusions as representative.

Of course it is possible that even such replicable phenomena may be of little theoretical importance; this is our second point. We feel that there is a regularity and a precision about these phenomena which suggest that nature is trying to tell us something; that these phenomena carry with them secrets about an understanding of learning, of memory, of the very way the brain behaves in processing and making use of new knowledge and skill. We believe that had we only the nous to read this coded message aright, we would be so much nearer an understanding of some of the central problems of psychology. Others may not share our enthusiasm, but we would beg them to stay with us to the end before making up their minds on this point.

Our third point reinforces this belief in the importance of pursuitrotor reminiscence and its attendant phenomena. Reminiscence interacts with other important areas of psychology in a precise, quantifiable, 
and theoretically meaningful manner. Motivation has important effects on reminiscence; high drive produces large reminiscence effects, low drive produces small ones. Yet, contrary to Hull's theory, high drive does not produce better pre-rest performance than low drive; such a finding is of great importance for any motivational theory. Personality, too, interacts with pursuit-rotor reminiscence; many studies agree in showing that extroverts demonstrate greater reminiscence than do introverts, although the two personality types do not show any difference in pre-rest practice. Even abnormal personality interacts with reminiscence, as Kraepelin (1895) posited long ago; schizophrenics show much less reminiscence than do normal or neurotic subjects. Lawful interactions such as these reinforce our belief that the whole set of phenomena (including also direct measures of arousal, and their interaction with reminiscence) is of great scientific importance, and cries out for a theoretical understanding which will serve to explain the observed facts in a parsimonious fashion.

We have chosen to discuss pursuit-rotor reminiscence because the various phenomena associated with this task can be demonstrated with great reliability and show regular and clear-cut relationships with basic task parameters, such as length of rest, and also important psychological concepts such as motivation and personality. Could we not, for similar reasons, pay more attention to reminiscence in other tasks as well? If by reminiscence we mean simply improvement in performance after rest, then we would seem to cast our net so wide that many heterogeneous phenomena would be thrown together; such improvement is seen in vigilance tasks (Stroh, 1972), in spiral after-effect (Holland, 1965), in eye-blink conditioning (Jones, 1974), in ergograph performance (Weiler, 1910), in work on Kraepelin's Rechenheft (Oehrn, 1895), in inverted alphabet printing (Kientzle, 1946), on the pathways test (C. H. Ammons, 1960), on a variety of motor skills tasks (Melton, 1947), and of course on verbal learning tasks as well (McGeoch \& Irion, 1952). We shall suggest that there are at least three different kinds of "reminiscence" involved in these various tasks, caused by quite different mechanisms. Ergograph-type tasks show "reminiscence" due to recovery from fatigue; vigilance-type tasks show "reminiscence" due to dissipation of inhibition; pursuit-rotor type tasks show "reminiscence" due to consolidation of learning. It would not be sensible to treat all these theoretically quite distinct effects of rest periods interpolated among periods of massed practice in the same book, or use the same term to characterize them; historically there has always been some confusion in the definition of the term "reminiscence," and we would suggest that there are good reasons to restrict its use to tasks involving only or mainly consoliclation-type processes in its causation. This means that 
the study of reminiscence would be concerned with the effects of rest on learning, rather than on performance; this is an important restriction which has in the past been followed by some writers, but not by others.

It will now be clearer why we have concentrated so much on just one of the many tasks which have been used in the study of reminiscence. As we shall argue, pursuit-rotor work is concerned in a relatively pure way with reminiscence as above conceived; there is little or no "fatigue" and little or no "inhibition" (in the sense of the word popularized by Ammons and Kimble in their classical studies of reminiscence). We started our work originally within the Hullian tradition, according to which pursuit-rotor reminiscence was explained in terms of dissipation of inhibition; after twenty years of experimental studies along these lines we feel that this explanation is definitely and definitively disconfirmed, and that some such explanation as have advanced in terms of consolidation is much nearer to the experimental facts. Whether this is so or not, the reader will be able to judge after perusing the pages of this book; we find it rather paradoxical that the one task which more than any other has been used to study, and to explicate hypotheses about, "reactive inhibition" and its dissipation, should be the one task which more than any other is completely free of any trace of inhibition and dissipation of inhibition.

We also believe that psychology may be able to learn an important lesson from the development of these theories, the changes that took place in them, and the final replacement of the inhibition theory by the consolidation theory; we hope to discuss these lessons in more detail in our last chapter. By tracing the development of investigations of a particular phenomenon over many years and also the rise and fall of the various theories devised to explain that phenomenon we hope that this book will be of interest not only to the student of motor skill learning, but also to all those actively engaged in practicing the art of experimental psychology.

It is considerations such as these which are responsible for the way this book was conceived and organized. Scientists are not usually very much interested in the history of their science; yet our theory would be difficult to understand without some rather detailed discussion of the development of the various conceptions which have been put forward to explain "reminiscence" and learning. As Medawar (1972, p. 105) has pointed out, "a scientist's present thoughts and actions are of necessity shaped by what others have done and thought before him; they are the wavefront of a continuous secular process in which The Past does not have a dignified independent existence of its own. Scientific understanding is the integral of a curve of learning; science therefore in some 
sense comprehends its history within itself." It seemed worthwhile to make the debt we owe to those who went before us more explicitparticularly as most textbooks fail to acknowledge the earliest experiments and theories in this field, which go back to Kraepelin and his students in the closing years of the last century. It is they who first discovered (explicitly) the phenomena of reminiscence, who advanced theories of inhibition and dissipation of inhibition to account for the fact, who discovered the phenomena of "blocking" (involuntary rest pauses) long before Bills, and who quantified many of the phenomena associated with reminiscence. It would be an interesting task for an historian to discover why their pioneering work was never given the recognition which it so richly deserves.

In line with our belief that history is important, and gives ballast to an otherwise purely factual and experimental account, we have given a somewhat detailed description, along historical lines, of the development of the experimental methods, and the apparatus, which eventually produced the classical work of Ammons and also of the theories which were developed to account for the facts discovered. Initially these theories were based on the concept of inhibition, but later this was abandoned in favor of consolidation.

The work of our Department mirrors clearly the change from inhibition to consolidation theory. We started out from the premises of Hullian theory, very much impressed by the apparent success of Ammons and Kimble to construct a theoretical system which appeared to cover all the observed phenomena. We tried to fit our own research findings into this system, and at first this process seemed to support the system in every detail. Predictions that extroverts would show greater reminiscence than introverts, or that high motivation would lead to greater reminiscence, were triumphantly verified. However, the worm was in the bud; there were many anomalous details, as we shall see in the course of this book, and attempts to shore up the leaning tower were of indifferent success. Finally it became clear that the whole system was becoming so complex and unwieldy, necessitating so many ad hoc liypotheses, that it ceased to be of any value in making predictions; it became like the Freudian opus, in that it could explain everything, and predict nothing-nothing, that is, that was not already known! Radical surgery was clearly required, and the consolidation theory was put forward as an alternative and less involved and complex hypothesis (Eysenck, 1965). This theory required much revamping and rethinking before it could be accepted as accounting for all the wellestablished research findings, and even the form presented here is still held only tentatively; we believe that it possesses the main advantage of 
a good theory in that it points to new and different types of research to those which we have become accustomed to. Whether the predictions which can be made from this new theory will continue to be borne out is of course a question for the future; it would be unwise to put too much faith in such a happy outcome. Indeed we are already aware of certain phenomena concerning the precise details of pursuit tracking performance which this theory cannot successfully account for. These are discussed in our chapter on "strategies."

Having outgrown our first love for inhibition theory, we still have lingering feelings of affection and regard for it. Although we have no doubt that it was completely inappropriate to pursuit-rotor learning and reminiscence, nevertheless it was a good theory in the proper scientific sense. For a time it accounted for the main facts in a satisfactory manner; it meshed with "big time theory" very adequately; and it made interesting and important predictions which could be experimentally tested and disproved. More than that no theory can be required to do, and it would be foolish to heap obloquy upon its remains, as many anti-Hullian theorists are apt to do. In retrospect, it is surprising how well an erroneous theory fitted the facts; how we seemed to be able to measure variables, such as $I_{R}$ and ${ }_{S} I_{R}$, which in fact do not enter the picture at all-certainly as far as pursuit-rotor learning is concerned. Such an experience must make one suspicious - when consolidation is evoked as an explanatory concept, are we fooling ourselves in a similar manner, only to find that thirty years later some new and untried concept will be advanced with equally high claims, and consolidation contemptuously dismissed? When such has been the fate even of apparently invincible concepts like Newton's universal gravitation, this seems only too likely. However, we cannot predict what kind of concept will take over in due course, and until anomalies accumulate and make such a change-over imperative, this is the best theory we have, and we suggest that it might be worthwhile trying it out to see how far it will get us.

It is the failure of the inhibition-type theories to produce an explanation of reminiscence which provided a major motivation for writing this book. We believe that pursuit-rotor learning was an ideal stamping ground on which to test conflicting theories about learning and memory. Our own theory grew as a reaction to the ever greater complexity of Hullian formulations, in their efforts to encompass the many findings which clearly did not fit in with the original hypotheses. The fifties and sixties provided a classical example of the picture given by Kuhn (1962) of the state of affairs preceding the scientific revolutions which he believes to occur whenever an existing theory (or paradigm) is encoun- 
tering too many anomolies which cannot be explained (or explained away) by modifications of the existing theory. In Lakatos's terms, a once progressive research programme shift has become regressive, more cuncerned with defence than with extension (Lakatos \& Musgrave, 1970), and it was time for a new research program to take its place. This was attempted in a programmatic fashion by Eysenck (1965), and we have tried to do so in a much more extensive and (we hope) successful manner in this book.

The need for such a venture, and an empirical analogy to the Kuhn and Lakatos interpretations of the history of scientific theory building, can be found in an interesting paper by Krantz (1965). He takes his departure from the results of counting the number of articles appearing each year in Physics and Chemistry Abstracts (Price, 1963); the yearly increase can be described by an exponential function. He then argues that a plotting of research activity in particular research areas within a science would show a large variety of curve forms, and asks the question "Can any consistency be found in the curve forms for research activity on this more molecular level of analysis?" Taking his cue from Kuhn, he argues that in the course of delineating the paradigm which constitutes current orthodoxy, research findings are obtained which violate the paradigm-induced expectations. "The course of 'anomalous' research is a more or less extended exploration of the area of the finding and ends when the anomaly has been shown to be either an unreliable event, assimilable into the current paradigm, or, least probably, produces a paradigm change where the finding is no longer perceived as contrary to expectation (scientific revolution). It would follow from this position that research in an 'anomalous' area should show a period of concentrated research, directed toward evaluating the finding, with a subsequent marked change in activity. The nature of this change would be dependent upon the outcome of the concentrated exploration; if the finding is shown to be unreliable or assimilable there should be a relatively rapid decrease in research with little probability of recovery of interest. However, if the findings eventuate in a paradigm change, the course of activity in the 'anomalous' area would show a continued and perhaps more accelerated increase in research. In short, it is hypothesized that a comparison between research activity in 'normal' vs. 'anomalous' areas should show differences primarily in the eventuation of research; in 'normal' science, activity should be generally continuous while 'anomalous' science should show either a marked decline with no recovery of interest, or a continuation of research." (Price, 1963, p. 39.)

In order to test this hypothesis, Krantz selected two cases of 'nor- 


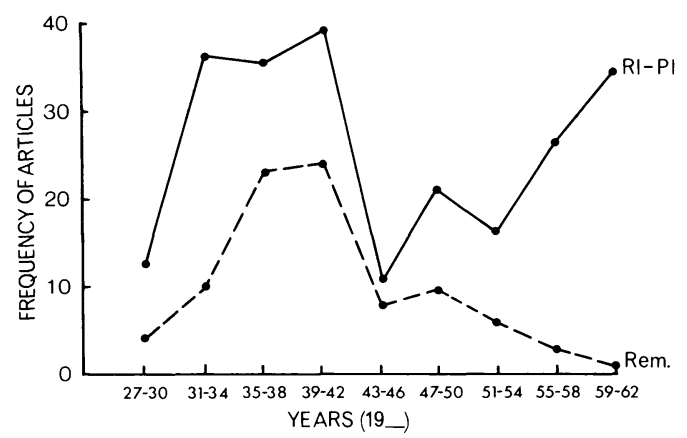

Figure 1. Research activity in verbal reminiscence and retroactive-proactive inhibition.

mal' and 'anomalous' research in psychology; "the 'anomalous' area is matched with a 'normal' area in that they represent explorations of similar viewpoints." One comparison was made between research activity in latent learning and secondary reinforcement; the other between activity in verbal reminiscence and retroactive-proactive inhibition. The frequency of articles, from 1927 to 1962, was found from consultation of the Psychological Abstracts, and the data for verbal reminiscence and retroactive-proactive inhibition are plotted in Figure 1. (A similar figure is plotted in Krantz's paper for latent learning and secondary reinforcement, showing latent learning research to have a similar course to verbal reminiscence, while secondary reinforcement had a similar course to retroactive and proactive inhibition.) "The research activity forms for the anomalous areas of latent learning and verbal reminiscence show very similar shapes; a rise in the frequency of research, followed by a period of high productivity, concentration or peaking and then a marked decline. The level of activity in the postdecline period is less than, or equal to, that prior to rise and concentration with no indication of subsequent recovery. It is clear, from the graphs and an analysis of the research findings in these 'anomalous' areas that a scientific revolution did not occur. Although it is difficult to pinpoint the factors contributing to decline in these two areas, an analysis of the literature indicates that latent learning was partially assimilated into the then current learning position (Hull, 1952) and for verbal reminiscence, accumulating evidence indicated that the phenomenon was unreliable (Hovland \& Kurtz, 1951)." (Krantz, 1965, p. 41.) "In contrast to the decline and lack of recovery in the 'anomalous' research curves, the activity in the 'normal' science areas shows a continual increase; secondary reinforcement presently in a period of increasing activity and retroactive-proactive inhibition recovering to its previous level after the 
World War II slump (the 1943-1946 point on the graph). In both areas a diminishing point in research is not yet apparent." (Krantz, 1965, p. 42.)

The data presented by Krantz are interesting, but his interpretation is somewhat doubtful. Motor reminiscence is one of the most reliable phenomena in experimental psychology, yet its course of research activity is not dissimilar to that of verbal reminiscence; here too we observe a period of high productivity following the work of Ammons and Kimble, to be replaced in recent years by a marked decline in the number of articles written. (The peak in research activity for motor reminiscence is some 10 to 15 years later than that for verbal reminiscence.) It is certainly true that verbal reminiscence is unreliable; Buxton (1943) called it the "now you see it, now you don't" phenomenon. But that may not be the only, or even the main reason for its decline; it may be as in the case of motor reminiscence, that the observed anomalies could not find an explanation within the context of Hullian learning theory. Unreliability undoubtedly aggravated the position, but it was itself probably produced by a failure to take into account variables which played no part in the traditional theoretical explanatory system of experimental psychology of the time (Eysenck, 1973). Clearly a "new look" was needed to replace a theoretical system which had run out of steam and could not account for the numerous anomalies which arose in the course of empirical work. We have attempted to provide such a "new look"; it will be interesting to see whether Krantz's prediction of a new rise in interest in the topic, following such a theoretical refurbishing, will be fulfilled.

It will be clear, from what has just been said, why we have concentrated on motor reminiscence, to the exclusion of most of the work that has been done on verbal reminiscence. Unreliable results make summary of findings meaningless, and do not lend themselves to theoretical conclusion; we have simply quoted in passing some studies indicating the importance of personality and motivation variables for a proper understanding of verbal reminiscence phenomena, and for their experimental control; beyond that it seemed inadvisable to go. A much more detailed treatment can be found in Eysenck (1973).

Another advantage of the broad historical approach we have chosen for our study of a rather specific phenomenon is that it enables us to discuss the advantages and disadvantages of various theoretical attitudes that psychologists have adopted.

Some critics will almost always oppose any kind of "conceptual nervous system" concept; they argue that such concepts are unnecessary, and that we should be contented with a set of differential equa- 
tions to describe our findings. Our theory can certainly be considered "dynamic" in the usual sense in which physicists use this term, i.e., expressible in terms of differential equations; we still believe, however, that psychological or physiological concepts, such as "consolidation" and "arousal," have an important part to play in such a theory. Similar arguments have of course gone on in physics too; as we shall see in the last chapter, the thermodynamic and kinetic theories of heat present a similar confrontation, the former dealing with unimaginable concepts of a purely quantitative kind, the latter giving us an eminently "visualizable" picture of what is going on when small particles in constant motion are colliding with each other (Eysenck, 1970). Perhaps a scientist's preference for one or other type of theory is determined by his extraverted or introverted tendencies; where physicists are still undecided about the respective value of these two ways of regarding nature, we would be hesitant to suggest any final conclusion.

Neither would we like to be dogmatic about another controversy which has frequently engaged experimental and theoretical psychologists, namely the debate about the relative usefulness of "big theory" or "small theory." Is it useful at the present time to try and put forward large-scale theories of the Hullian type, or should we rather occupy ourselves with "miniature theories," relating to a particular phenomenon, or even a particular phenomenon as demonstrated on a particular piece of apparatus? To ask the question is to realize that fundamentally it is meaningless. The reaction to Hull's great system, and its relative failure, has been exaggerated; we need fundamental concepts, and general theories, just as much as we need specific applications, in great detail, to selected areas. Our book deals with a very limited area, reminiscence in pursuit-rotor learning, but the concepts we find necessary (consolidation, extraversion-introversion, motivation) are obviously of much greater latitude, and would apply to many other areas. We find this whole discussion unreal; Hull's theories resembled the curate's egg, which means that they were neither wholly good nor wholly bad, although the rapid swing of opinion would suggest that they were the former at one time, and are the latter now. This is an unrealistic way of looking at theories as if they were modish changes in skirt length. What Hull tried to do will have to be done sometime; it seems slightly absurd to throw out the baby with the bath water. But of course if theories au grand do not fit the facts, even in a small backwater like pursuit-rotor reminiscence, they have to go; there is a reciprocal relation between "big theory" and "small theory" which advocates of either may not always recognize. 
As expressed in our dedication of this book to the large number of students and colleagues who have worked in this Department, on problems associated with reminiscence, we have based ourselves very much on the principles expressed in some detail in an article on "Programme research and training in research methodology" (Eysenck, 1970). Seeing a problem in science from a broad, historical perspective is of importance not only for understanding particular theories and how they have developed, but also for discerning the line of future research that is likely to be productive. It follows from this belief that training in research can best be accomplished by having the student work on a kind of apprenticeship level with an experienced research worker, on problems which have engaged the Department for a long time. Participation in such a program eliminates the "one off" type of research so familiar in psychology; research which is inherently unlikely to produce worthwhile results, which ends just when the student is in a position to make a proper start, and which is never followed up by anyone else-in spite of the traditional last words about further research being required. Program research shows the student the value of continued work on a particular set of problems, it makes certain that his work and results will indeed be followed up by the next generation of students, and it shows him how his own work is based intimately on that of his predecessors. These are important insights for a research worker to gain; he is more likely to make them his own when experience shows their value, rather than when he simply reads about them in some text book or other. Our experience suggests that these theoretical propositions possess some value for the training of research workers in psychology; whether the accumulated work done by generations of students on the problems discussed in this book has been worth the trouble must be left to the reader to judge.

Institute of Psychiatry

University of London

H. J. EYSENCK

C. D. FRITH 